\title{
Ventricular androgenic-anabolic steroid-related remodeling: an immunohistochemical study
}

\author{
Rossana Cecchi $^{1}$ (D) Barbara Muciaccia $^{2}$ - Costantino Ciallella ${ }^{2}$. \\ Natale Mario Di Luca ${ }^{2}$ - Akihiko Kimura ${ }^{3}$. Cristina Sestili ${ }^{4}$ Mizuho Nosaka ${ }^{2}$. \\ Toshikazu Kondo ${ }^{1}$
}

Received: 12 September 2016 / Accepted: 4 April 2017

(C) Springer-Verlag Berlin Heidelberg 2017

\begin{abstract}
Background Several fatal cases of bodybuilders, following a myocardial infarction after long exposure to androgenicanabolic steroids (AAS), are reported. In recent years, evidence has emerged of cases of heart failure related to AAS consumption, with no signs of coronary or aorta atherosclerosis. This study aims to further investigate the pathogenesis of the ventricular AAS-related remodeling performing immunohistochemistry (IHC).

Method In order to examine innate immunity activity and myocytes and endothelial cell apoptosis, IHC analyses were performed on heart tissue of two cases of bodybuilders who died after years of supratherapeutic use of metelonone and nandrolone and where no atherosclerosis or thrombosis were found, using the following antibodies: anti-CD68, anti-iNOS, anti-CD163, anti-CD 15, anti-CD8, anti-CD4, anti-HIF1 $\alpha$, and in situ TUNEL staining.

Results Results confirm the experimental findings of recent research that, in the absence of other pathological factors, if intensive training is combined with AAS abuse, myocytes and endothelial cells undergo apoptotic alterations. The absence of
\end{abstract}

Rossana Cecchi

rossana.cecchi@unipr.it

1 Department of Medicine and Surgery, Via Gramsci 14, 43126 Parma, Italy

2 Department of anatomical, histological, medico-legal and orthopedic sciences, Section of Histology, Sapienza University of Rome, Rome, Italy

3 Department of Forensic Medicine, Institute of Advanced Medicine, Wakayama Medical University, Wakayama, Japan

4 Department of Public Health, Sapienza University of Rome, Rome, Italy inflammatory reactions and the presence of an increased number of M2 macrophages in the areas of fibrotic remodeling confirm that the fibrotic changes in the heart are apoptosisrelated and not necrosis-related.

Conclusions In conclusion, the study indicates that, in very young subjects with chronic hypoxia-related alterations of the heart, signs of a heart failure in the other organs and a history of AAS abuse, death can be ascribed to progressive heart failure due to the direct apoptotic cardiac and endothelial changes produced by AAS.

Keywords Androgenic-anabolic steroids · Autopsy · Death · Heart failure · Apoptosis $\cdot$ Chronic hypoxic changes ·

Myocardial remodeling

\section{Introduction}

Doping is the use of drugs to improve athletic performance. It is prohibited by anti-doping rules, and a list of the drugs considered illicit, called The List, is yearly updated by the World Anti-Doping Agency (WADA).

At present, $48 \%$ of all positive doping cases still involves androgenic-anabolic steroids (AAS) as noted by the WADA in 2014 [1]. Doses 100-1000 times in excess of the therapeutic range of AASs are commonly used [2]. Side effects are seen in individuals using or having used high-dosage androgen without preceding androgen deficiency. The complications are mainly non-fatal; however, they may lead to severe consequences involving cerebral thrombosis or cardiac infarction [3-5]. Few fatal cases of bodybuilders, following a myocardial infarction after long exposure to AAS, are reported [6,7]. These subjects often suffer from hyperlipidemia, hypercholesterolemia, and precocious atherosclerosis secondary to AAS consumption in supraphysiological doses. Therapeutic uses of AAS are showing 
improvements in aging males for physiologic testosterone replacement and as an antianginal in cardiac patients. Therefore, the correct dose to be used to prevent adverse effects has to be clarified [8].

In recent years, evidence has emerged of cases with heart failure, but no signs of coronary or aorta atherosclerosis where the most important pathological findings have been found, not only in gross examination but more frequently in histological samples [9-13].

To further investigate the effects of AAS abuse on myocardial tissue, and the pathogenesis of the ventricular AASrelated remodeling, we present two cases of bodybuilders who died after years of supratherapeutic use of metelonone and nandrolone and where no atherosclerosis or thrombosis was found. Innate immunity activity and myocytes and endothelial cell apoptosis, which are related to the ventricular remodeling as discussed further, were studied histologically on the myocardium by performing immunohistochemistry (IHC).

\section{Case reports}

\section{Case 1}

A 20-year-old bodybuilder, taken ill early in the morning, died before reaching hospital. Empty packages of Primobolan Depot (metelonone enantate) and Dynabolon (nandrolone undecylenate) were found in his apartment. According to friends of the deceased, he had been taking AAS for a few years before his death.

Body weight was $87 \mathrm{~kg}$, height $190 \mathrm{~cm}$, and body mass index (BMI) 28.41.

The post-mortem examination revealed that he had conspicuously well-developed skeletal musculature, a low percentage of subcutaneous fat tissue, and intense nail cyanosis of the upper limbs and the ear auricles.

On internal examination, there were no findings suggesting mechanical trauma or asphyxiation; but the brain and lungs were heavily congested; the lungs with subpleural petechiae; and the liver, spleen and kidneys were congested. The heart showed cardiomegaly (440 g), with hypertrophy and biventricular dilatation (21 mm ventricular septum, $19 \mathrm{~mm}$ left ventricular free wall, $5 \mathrm{~mm}$ right ventricular free wall). Atrioventricular and semilunar valves were normal. The coronary arteries had a normal origin and course and were patent. The histological examination revealed, at the interventricular septum, a hypertrophy of the myocardial fibers, some groups of elongated and thinned fibers, widespread interstitial and mainly perivascular fibrosis, hyaline degeneration, and cell damage. The left ventricle showed extensive focal disarray with interstitial and replacement fibrosis and widespread interstitial edema; the subendocardial trabecolae showed areas of colliquative myocytolysis, replacement-type fibrosis, and extensive perivascular fibrosis, resulting in patches of small to medium-sized scar areas, areas of fibrofatty replacement, and a few foci of neovascularization with some instances of plugged capillaries (Fig. 1a).

The liver showed cholestasis and steatosis. The lungs were heavily congested, and all lobes revealed multiple areas of erythrocyte containing alveoli and areas of emphysema alternated with areas of atelectasis. There was intensive congestion of the kidneys, adrenals, and thyroid.

A chemical-toxicological analysis searching for doping substances and drug abuse were performed with GC-MS/MS and showed the presence of AAS in the deceased's urine (nandrolone $5 \mathrm{ng} / \mathrm{ml}$, metelonone $(7 \mathrm{ng} / \mathrm{ml})$.

\section{Case 2}

A 23-year-old bodybuilder was found dead in his bed.

According to friends and parents, he had been taking AAS for 2 years before his death and his muscle mass had increased by about $14 \mathrm{~kg}$.

The body showed hypertrophy of the skeletal musculature. He was $175 \mathrm{~cm}$ tall and weighed $106 \mathrm{~kg}$, with a BMI of 34.61 .

The autopsy revealed that the heart showed cardiomegaly (430 g), with concentric left ventricular hypertrophy (22 mm ventricular septum, $20 \mathrm{~mm}$ left ventricular free wall, $7 \mathrm{~mm}$ right ventricular free wall). Atrioventricular and semilunar valves were normal. The coronary arteries had a normal origin and course and were patent. No signs of atherosclerosis were found in the aorta. The lungs and liver were heavily congested.

The histologic exam revealed hypertrophied myocytes on the left ventricle and the interventricular sept, with minimal myocardial necrosis as seen from the few small foci of granulation tissue and the extensive interstitial, perivascular, and subendocardial fibrosis (Fig. 2a). No gross and histologic alterations of the other organs were seen, except for congestion.

The chemical-toxicological analysis searching for doping substances and drug abuse were performed with GC-MS/MS and showed the presence of AAS in the deceased's urine (nandrolone $7 \mathrm{ng} / \mathrm{ml})$.

\section{Immunohistochemical and in situ TUNEL analyses}

\section{Materials and methods}

In order to examine innate immunity activity and myocytes and endothelial cell apoptosis, IHC analyses were performed on heart tissue using the following antibodies: anti-CD68 for macrophages (DAKO, Carpinteria, CA), anti-iNOS for M1 macrophages (Abcam, Tokyo, Japan), anti-CD163 for M2 macrophages (Abcam), anti-CD 15 for neutrophils (DAKO), 


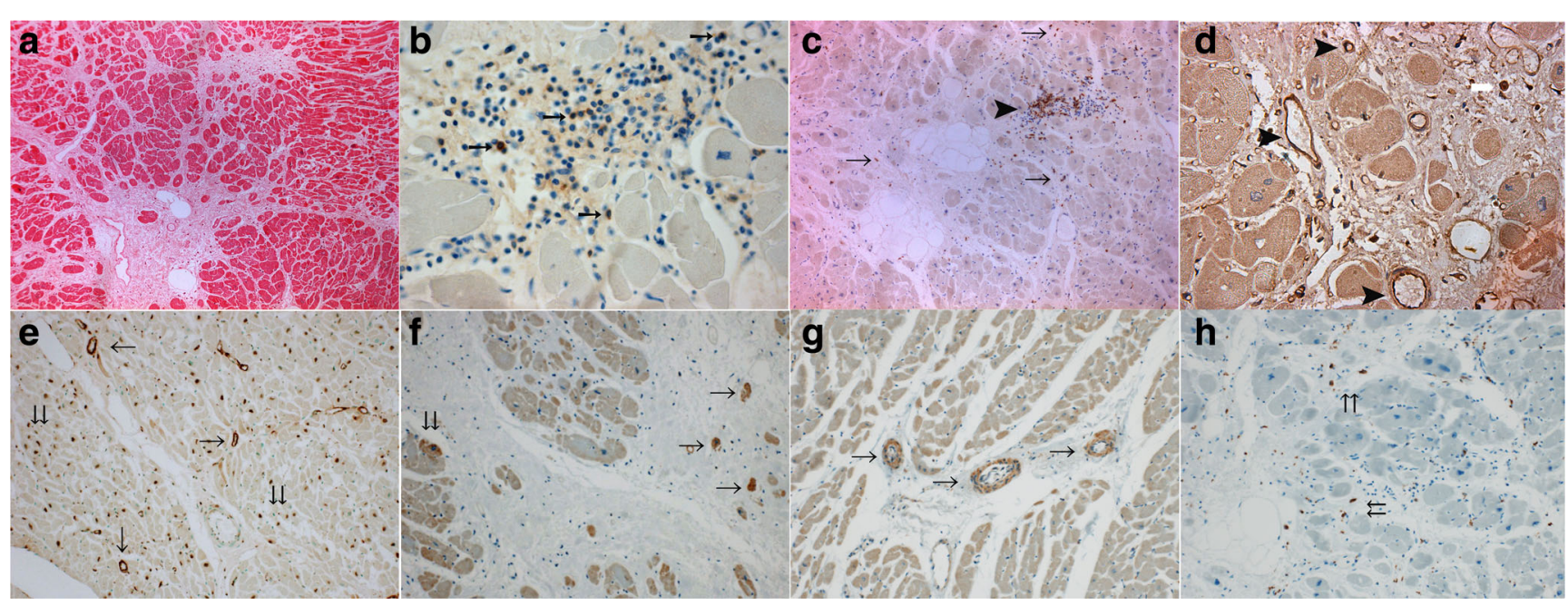

Fig. 1 Heart tissue of case 1. a Left ventricle showing interstitial and replacement fibrosis resulting in patches of small to medium-sized scar areas, areas of fibrofatty replacement, and a few foci of neovascularization with some instances of plugged capillaries (H.E. 10×). b A focal inflammatory infiltrate with few CD8+ lymphocytes (arrows) (antiCD8, 40×). c Several macrophages CD68+ throughout the sample (arrows) and in an inflammatory infiltrate (arrowhead) (anti-CD68, $10 \times$ ). d Anti-HIF1- $\alpha+$ endothelial cells in vessels (arrowheads) and

anti-CD8 (DAKO), anti-CD4 (DAKO), and anti-HIF1 $\alpha$ (clone EE122and anti-caspase 3 (Cell Signaling Technology, Tokyo, Japan). Moreover, in situ TUNEL staining (ApopTag leucocytes (white arrow) in an area of fibrotic replacement (anti-HIF1$\alpha, 10 \times$ ). e Foci of TUNEL-positive-stained myocytes (double arrows) and endothelial cells of many vessels (arrows) (in situ TUNEL staining, $10 \times$ ). f Caspase $3+$ myocardial cells (arrows) in areas undergoing fibrotic changes and in normal heart tissue (double arrows) (anti-caspase $3,10 \times$ ). g Caspase 3+ endothelial and muscular cells of vessels (arrows) (anticaspase 3,10×). h Anti-CD 163 staining shows several M2-type macrophages (double arrows) (anti-CD 163, 20×)

Peroxidase In Situ Apoptosis Detection Kit, Millipore, Temecula, CA) was also carried out for the detection of apoptosis.

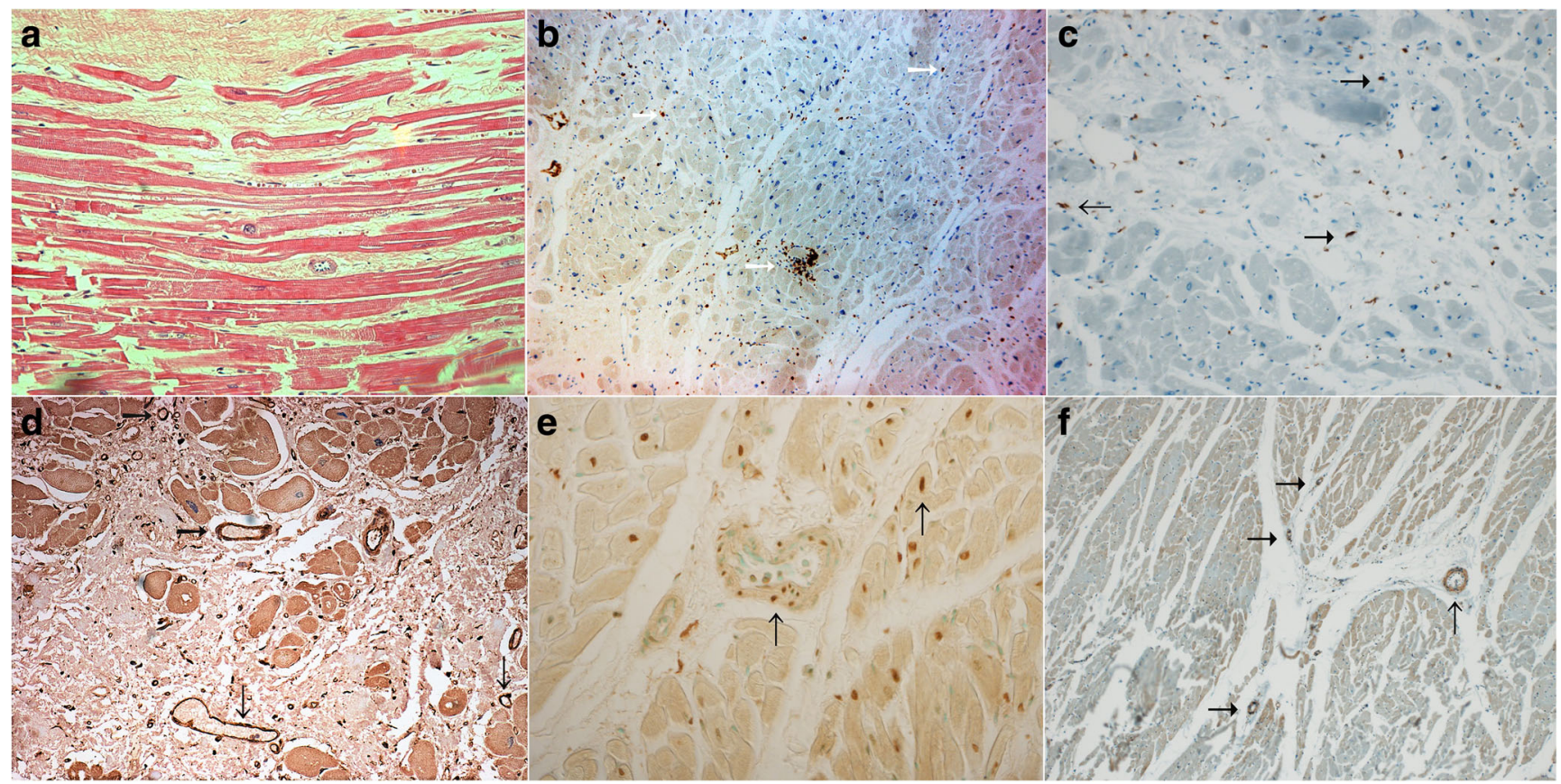

Fig. 2 Heart tissue of case 2. a Hypertrophied myocytes of the left ventricle of case 2 and extensive interstitial, perivascular, and subendocardial fibrosis (H.E. 20×). b Anti-CD 68-positive macrophages throughout the heart tissue and in a focal inflammatory infiltrate (arrows) (anti-CD 68, 10×). c Anti-CD 163-positive M2-type macrophages throughout the heart tissue (arrows) (anti-CD 163, 10×). d Anti-HIF1$\alpha$-positive endothelial cells in several vessels throughout the sample (arrows), particularly in the areas of fibrotic replacement (anti-HIF- $\alpha$, $20 \times$ ). e TUNEL-positive-stained myocytes (arrow) and positive-stained endothelial and muscular cells of some arterial vessels (arrow) in a nonfibrotic areas (TUNEL, 20×). f Caspase 3 positive prevalently in the endothelial cells of many small-sized vessels (arrows) (anti-caspase 3, $10 \times)$ 


\section{Case 1}

IHC staining showed rare focal inflammatory infiltrates with some CD8+ lymphocytes and several macrophages CD68+ (Fig. 1b, c), while CD15+ neutrophils were scattered in the myocardium without forming infiltrates, and CD4 lymphocytes were absent. Anti-HIF1- $\alpha$-positive endothelial cells were found in several vessels of different sizes throughout the sample, particularly in the areas of fibrotic replacement (Fig. 1d). TUNEL staining revealed throughout foci of positive-stained myocytes and positivity of endothelial cells of many vessels (Fig. 1e). In the areas undergoing myocyte replacement with fibrotic tissue, vessels showed only some of the endothelial cells in apoptosis. In the fibrotic areas, no vessels stained positive, and damaged myocytes were positive as well as some fibroblasts and monocytes. Caspase 3 stained positive in many foci of myocardial cells, especially in areas undergoing fibrotic changes (Fig. 1f) and in endothelial and muscular cells of several vessels of different sizes (Fig. 1g). Purkinje cells also stained positive with caspase 3. CD68+ macrophages were present throughout the tissue, increasing in the areas undergoing fibrotic replacement, whereas in some areas, focal microinfiltrates were identifiable. Anti-iNOS and anti-CD 163 staining showed that all macrophages where M2type (Fig. 1h), while only rare or none M1-type macrophage was identifiable.

A sample of deltoid muscle was stained following the same procedure and showed positivity only to caspase 3 and TUNEL of endothelial and muscular cells of several arteries.

\section{Case 2}

Immunostaining showed few focal inflammatory infiltrates formed by lymphocytes and CD68+ macrophages (Fig. 2b). CD68+ cells were present throughout the tissue, and anti-CD 163 staining proved them to be all M2 macrophages (Fig. 2c), while rare M1 i-NOS+ cells were found in the epicardium and interstitial tissue. CD15+ neutrophils were scattered in the myocardium without forming infiltrates, and CD4+ lymphocytes were absent. Anti-HIF1- $\alpha$-positive endothelial cells were found in several vessels throughout the sample, particularly in the areas of fibrotic replacement (Fig. 2d). TUNEL staining revealed throughout foci of positive-stained myocytes and positivity of endothelial and muscular cells of some different-sized arterial vessels, less in number than in case 1, especially in the non-fibrotic areas (Fig. 2e). Caspase 3 was positive prevalently in the endothelial cells of many small-sized vessels, and in several cells of the epicardium and interstitial tissue, and in the Purkinje cells (Fig. 2f).

\section{Control case}

The cardiac tissue taken from a 16-year-old youth who had died after a fall from the height was analyzed as a control case. The weight of the heart was $220 \mathrm{~g}$, and there was neither macroscopic anomaly nor injury. No histopathological alterations were found. At the IHC, no presence of CD15+ neutrophils was shown, while CD68+ cells were present in all the tissue and were identified as M2 macrophages without forming infiltrates (Fig. 3a). No M1 macrophages and no T lymphocytes were found. TUNEL documented positive myocytes in the subendocardium, rare myocytes in the myocardium, and several cells in the interstitium. Vessel walls showed no positivity with TUNEL staining. Caspase 3 was positive in several cells in the subendocardium tissue and in a few small vessels and in the Purkinje cells. Positive reactions for caspase 3 were found in the few myocardiocytes (Fig. 3b).

\section{Discussion}

It is of common opinion that cardiac hypertrophy resulting solely from physical exercise has minimal or no harmful effects on the heart. This is true even for the most strenuous of sports. In fact, a non-AAS user athlete who dies due to heart trouble usually had a pre-existing undiagnosed cardiomyopathy [14]. Most persons who grossly misuse AAS by stacking with supra-therapeutic doses are bodybuilders although some weightlifters especially powerlifters may do so. None of these persons engage in endurance training which is commonly associated with physiological, i.e. non-pathological cardiac hypertrophy. Certainly when engaging in weight training, these persons can and often do experience hypoxia especially if they unwisely breath-hold. Hence, such training should not cause physiological cardiac hypertrophy and if it occurs as in the two cases described, it seems reasonable to suggest it is a direct consequence of administering supra-therapeutic doses of AAS over an extended period of time and could be considered to be "non-physiological."

Cardiovascular implications of supraphysiological androgen levels still require further clarification, and concentric left ventricular hypertrophy is being studied by means of imaging techniques on living athletes. It has confirmed that use of AAS may lead to cardiac hypertrophy and has hypothesized a direct cardiotropic effect [15] associated with a significantly lower left ventricle ejection fraction and decreased diastolic function [16] strongly associated with mean dosage and duration [17, 18]. Furthermore, some recent innovative diagnostic techniques, such as pulsed-wave tissue Doppler imaging and ultrasonic-integrated backscatter cyclic variation analysis [19] and cardiac magnetic resonance (CMR) [20], documented regional systolic and diastolic dysfunction in AAS users with respect to AAS non-users or control cases. Angell and 


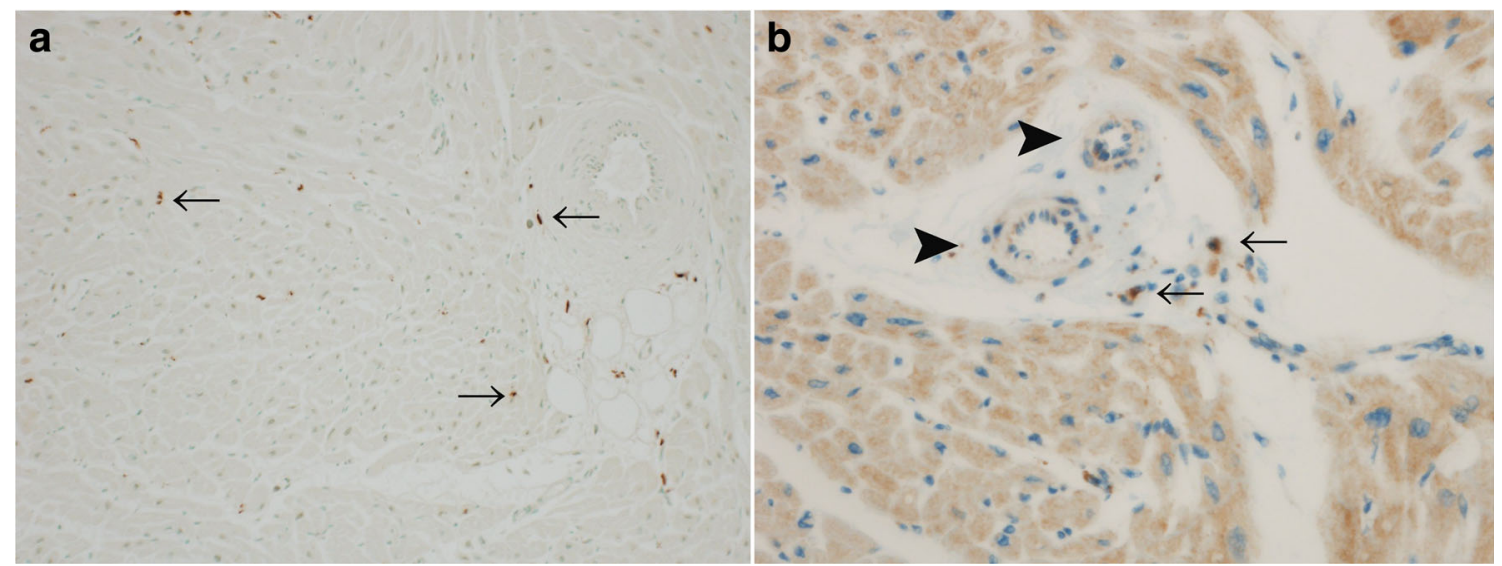

Fig. 3 Heart tissue of the control case. a Anti-CD 163-positive M2-type macrophages sparsely in the tissue without forming infiltrates (arrows). b Caspase 3-negative endothelial cells in two small vessels (arrowheads) and positive in few apoptotic myocardocytes (arrows) (anti-caspase 3, 20×)

colleagues [21] could detect pathological alterations of ventricular structure and dysfunction in the hearts of AAS users in CMR analyses. However, focal cardiac fibrosis, which is almost found histopathologically in AAS users, could not be detected by the use of CMR. Considering from these observations, CMR analyses would be insufficient for the detection of focal cardiac fibrosis.

In our study, the morphological features found in the heart tissue of the cases presented agree with most of those found in similar cases of death due to anabolic abuse. There are no signs of atherosclerosis or thrombosis, i.e. focal myocardial fibrosis, but with foci of contraction band necrosis [9], concentric cardiac hypertrophy with focal fibrosis, dilated cardiomyopathy with patchy myocyte death, eosinophilic myocarditis [10], few small foci of granulation tissue and lymphocytic infiltration around vessels [11], marked cardiac and renal hypertrophy and hepatosplenomegaly, with regional myocardial fibrosis and focal myocardial necrosis [12], and acute cellular necrosis and interstitial fibrosis of the myocardium [13].

Moreover, the IHC staining performed (caspase 3 and TUNEL staining) also shows a significant increase in the rate of cardiomyocyte and endothelial cell apoptosis in AAS abusers. This feature is common, not only in acute ischemic hearts, where caspase 9 activation triggers cardiomyocyte and endothelial cell apoptosis [22], but also in dilated cardiomyopathy and heart failure. In these cases, the apoptotic loss of cardiomyocytes plays an important role in the speed of heart failure progression [23].This hypothesis has been confirmed by an experimental study that showed elevated caspase 3 activity in the hearts of AAS-treated rabbits compared to control experiments, suggesting that apoptosis is involved in the induction of norethandrolone-induced cardiac lesions [24]. Moreover, there is evidence of increased cardiomyocyte apoptosis following the transition from compensated hypertrophy to left ventricle dysfunction [25].
Although caspase 3 is the terminal effector caspase for both intrinsic (mitochondrial-dependent) and extrinsic (death receptor ligand-dependent) apoptosis pathways, several studies underline the important role of mitochondrial pathway in the apoptosis AAS-related. It is well known that both physiological cell death (apoptosis), and, in some cases, accidental cell death (necrosis), relies on the liberation of apoptogenic proteases or protease activators from mitochondria [26]. In sedentary rats treated with anabolic steroids, hypertrophy and mitochondria fusion occur, resulting in an increase in volume and a decrease in mitochondria numbers, whereas the production of ATP remains the same, thus being proportionally insufficient for the needs of the hypertrophic cell. A combination of training and anabolic steroid use destroys the mitochondria triggering a caspase cascade that leads to cell death by apoptosis $[27,28,29]$. The loss of production in cellular energy can result in a chronic hypoxia of the cardiac muscle, also favored by the coronary-spastic action of the steroids. In these cases, cell death may occur due to apoptosis, rather than necrosis.

Genetics may also be responsible for some mitochondrial reactions to training and AAS and could explain why AAS abusers react in many different ways - some being unaffected, others only with complications, and others dying [30, 31].

During a steady state, macrophages in the heart show a gene profiling of M2 and carry out routine repairs and maintain tissue integrity. Also, M1 macrophages direct T cells toward Th1-, and M2 macrophages toward Th2-like activities and are dominant in the first stage of heart injury, i.e. infarction $[32,33]$. In the two cases presented, compared to the control case, an increased number of macrophages CD68+, proven to be mature macrophages (M2 differentiation stage), especially in the myocardium and in focal infiltrations in the myocardium undergoing fibrosis, were documented, whereas in the first maturation stage, M1-type macrophages were absent, indicating that there is no active inflammatory response. 
This feature is found in ischemic hearts in a regenerative phase, in which cells with a lesser inflammatory phenotype promoting tissue repair of M2-type macrophages prevail, and in idiopathic dilated cardiomyopathy, as well as in animal models of heart failure and remodeling [25].

As well known, apoptosis does not result in an inflammatory stimulus, and this could explain the presence, in our cases, of CD 15+ neutrophils only scattered throughout the myocardial tissue, while the presence of few lymphocytes CD8+ in some foci of macrophages could be tied to their activity as damaged cell phagocytes. The high number of M2 macrophages throughout the myocardium, and in foci in the damaged tissue, necessary for the fagocitation of apoptotic cells, contributes to the theory of a progressive loss of myocytes through apoptosis, and through the macrophage production of stress-activated cytokines that leads to progressive heart failure [25].

An in vitro study revealed an apoptotic steroid- type-dependent effect in human umbilical vein endothelial cells (testosterone induced $31 \%$ of apoptotic cells, norandrostenedione $25 \%$, androstenedione $15 \%$, and nandrolone $18 \%$ ) and showed that androgen steroids modulate intracellular levels of calcium, independent of incubation time or compound identity, through the alteration of the endothelial homeostasis, which facilitates early endothelial cell activation responsible for vascular complications observed frequently in AAS users [34]. Our results confirm the detrimental action that AAS exerts on tissue vessel walls. The cardiac tissue of the two AAS abuse cases, compared to the control case, showed apoptotic reaction, as shown by TUNEL and anti-caspase 3 , in several vessel endothelial and muscular cells, as well as in the muscle sample taken in case 1 .

\section{Conclusion}

There are many difficulties in interpreting the experimental data from animals (mice and rats) related to AAS abuse, due to the diversity of substances used, the different sex and age of the mice/rats, the AAS treatment times, dosages used, type, scope, and exercise duration [35]. Therefore, verifying these results in the case of humans is of paramount importance, since the pathophysiological mechanism of AAS-related deaths is still obscure in several aspects [36].

In the cases presented, the microscopic findings in the cardiac tissue confirm the experimental findings of recent research that, in the absence of other pathological factors, if intensive training is combined with AAS abuse, myocytes and endothelial cells undergo apoptotic alterations, which are especially related to mitochondrial damage, as documented by experimental researches. The ATP-related loss of energy in myocytes and endothelial cells, and their apoptotic changes, causes a chronic hypoxia-like condition that leads to the progressive fibrotic remodeling of cardiac tissue. The absence of inflammatory reactions, as seen by the immunostainings performed, and the presence of an increased number of M2 macrophages in the areas of fibrotic remodeling confirm that the fibrotic changes in the heart are apoptosis-related and not necrosis-related.

In conclusion, although it concerns only two cases, our study documents the findings so far showed in experimental studies, that, in very young subjects with no history of chronic systemic pathologies, no signs of atherosclerosis, but with chronic hypoxia-related alterations of the heart, signs of a heart failure in the other organs and a history of AAS abuse, death can be ascribed to progressive heart failure due to the direct apoptotic cardiac and endothelial changes produced by AAS. As far as we know, this is the first immunohistochemical study on this topic. Obviously, further studies on human cardiac tissue in doping cases and controls are needed, which we encourage for a comparison with ours.

Acknowledgements Authors acknowledge Noemi Panebianco and Emi Shimada for their outstanding technical work.

Compliance with ethical standards The histological staining was performed on human specimens used for forensic reasons.

Conflict of interest The authors declare that they have no conflict of interest.

\section{References}

1. World Anti-Doping Agency (2014) Anti-doping testing figures report. www.wada-ama.org

2. Parkinson AB, Evans NA (2008) Anabolic androgenic steroids: a survey of 500 users. Med Sci Sports Exer 38:644-651

3. Sullivan ML, Martinez CM, Gennis P, Gallagher EJ (1998) The cardiac toxicity of anabolic steroids. Prog Cardiovasc Dis 41:1-15

4. Madea B, Grellner W (1998) Long-term cardiovascular effects of anabolic steroids. Lancet 352:33

5. Madea B, Grellner W, Musshoff F, Dettmeyer R (1998) Medicolegal aspects of doping. J Clin Forensic Med 5(1):1-7

6. McNutt RA, Ferenchick GS, Kirlin PC, Hamlin NJ (1988) Acute myocardial infarction in a 22 -year-old world class weight lifter using anabolic steroids. Am J Cardiol 62:164

7. Ferenchick GS, Adelman S (1992) Myocardial infarction associated with anabolic steroid use in a previously healthy 37-year-old weight lifter. Am Heart J 124:507-508

8. Papamitsou T, Barlagiannis D, Papaliagkas V, Kotanidou E, Dermentzopoulou-Theodoridou M (2011) Testosterone-induced hypertrophy, fibrosis and apoptosis of cardiac cells - an ultrastructural and immunohistochemical study. Med Sci Monit 17:266-273

9. Fineschi V, Baroldi G, Monciotti F, Pagliacci Reattelli L, Turillazzi E (2001) Anabolic steroid abuse and cardiac sudden death. Arch Pathos Lab Med 125:253-255

10. Montisci M, El Mazloum R, Cecchetto G, Terranova C, Ferrara SD, Thiene G, Basso C (2012) Anabolic androgenic steroids abuse and cardiac death in athletes: morphological and toxicological findings in four fatal cases. Forensic Sci Int 217:1-3 
11. Thiblin I, Mobini-Far H, Frisk M (2009) Sudden unexpected death in a female fitness athlete with a possible connection to the use of anabolic androgenic steroids (AAS) and ephedrine. Forensic Sci Int 184:1-3

12. Luke JL, Farb A, Virmani R, Sample RHB (1990) Sudden cardiac death during exercise in a weight lifter using anabolic androgenic steroids: pathological and toxicological findings. J Forensic Sci 35: 1441-1447

13. Hausmann R, Hammer S, Betz P (1998) Performance enhancing drugs (doping agents) and sudden death - a case report and review of the literature. Int J Legal Med 111:261-264

14. Robbins SL, Cotran RS (2015) In: Kumar, Abbas, Aster (eds) Pathologic basis of disease, Ninth edn. Elsevier, Amsterdam

15. Mobini Far HZ, Ågren G, Thiblin I (2012) Cardiac hypertrophy in deceased users of anabolic androgenic steroids: an investigation of autopsy findings. Cardiovasc Pathol 21(4):312-316

16. Baggish AL, Weiner RB, Kanayama G, Hudson JI, Picard MH, Hutter AM, Pope Long HG (2010) Term anabolic-androgenic steroid use is associated with left ventricular dysfunction. Circ Heart Fail 3:472-476

17. D'Andrea A, Caso P, Salerno G, Scarafile R, De Corato G, Mita C, Di Salvo G, Severino S, CuomoS LB, Esposito N, Calabro R (2007) Left ventricular early myocardial dysfunction after chronic misuse of anabolic androgenic steroids: a Doppler myocardial and strain imaging analysis. Br J Sports Med 41:149-155

18. Zaugg M, Jamali NZ, Lucchinetti E, Xu W, Alam M, Shafiq SA, Siddiqui MAQ (2001) Anabolic-androgenic steroids induce apoptotic cell death in adult rat ventricular myocytes. J Cell Physiol 187:90-95

19. Montisci R, Cecchetto G, Ruscazio M, Snenghi R, Portale A,Viel G, Nalesso A, Paoli A, Illiceto S, Meloni L, Ferrara SD, Montisci M (2010) Early myocardial dysfunction after chronic use of anabolic androgenic steroids: combined pulsed-wave tissue doppler imaging and ultrasonic integrated backscatter cyclic variations analysis. J Am Soc Echocardiogr 23:516-522

20. Luijkx T, Velthuis BK, Backx FJ, Buckens CF, Prakken NH, Rienks R, Mali WP, Cramer MJ (2013) Anabolic androgenic steroid use is associated with ventricular dysfunction on cardiac MRI in strength trained athletes. Int J Cardiol 167:664-8

21. Angell PJ, Ismail TF, Jabbour A, Smith G, Dahl A, Wage R, Whyte G, Green DJ, Prasad S, George K (2014) Ventricular structure, function, and focal fibrosis in anabolic steroid users: a CMR study. Eur J Appl Physiol 114:921-8

22. Scarabelli TM, Stephanou A, Pasini E, Comini L, Raddino R, Knight RA, Latchman DS (2002) Different Signaling Pathways Induce Apoptosis in Endothelial Cells and Cardiac Myocytes During Ischemia/Reperfusion Injury. Circulation Research 90: 745-748
23. Saraste A, Pulkki K, Kallajoki M, Heikkilä P, Laine P, Mattila S, Nieminen MS, Parvinen M, Voipio-Pulkki LM (1990) Cardiomyocyte apoptosis and progression of heart failure to transplantation. Eur J Clin Invest 9:380-386

24. Fantona L, Belhanib D, Vaillantb F, Tabiba A, Gomezc L, Descotesd J, Dehinab L, Bui-Xuanb B, Maliciera D, Timourb Q (2009) Heart lesions associated with anabolic steroid abuse: comparison of post-mortem findings in athletes and norethandroloneinduced lesions in rabbits. Exp Toxicol Pathol 61:317-323

25. Condorelli G, Roncarati R, Ross J, Pisani A, Stassi G, Todaro M, Trochai S, Drusco A, Gu Y, Russo MA, Frati G, Jonesi SP, Leferi DJ, Napoli C, Croce CM (2001) Heart-targeted overexpression of Caspase 3 in mice increases infarct size and depresses cardiac function. PNAS 98:9977-9982

26. Kroemer G, Dallaporta B, Resche-Rigon M (1998) The mitochondrial death/life regulator in apoptosis and necrosis. Annu Rev Physiol 60:619-642

27. Dragovich T, Rudin CM, Thompson CB (1988) Signal transduction pathways that regulate cell survival and cell death. Oncogene 17: 3207-3213

28. Nuñez G, Benedict MA, Hu Y, Inohara N (1998) Caspases: the proteases of the apoptotic pathway. Oncogene 17:3237-3245

29. Green DR, Reed JC (1998) Mitochondria and apoptosis. Science 281:1309-1312

30. Satoh K, Gotoh T, Yamashita K (2000) Morphological effects of an anabolic steroid on muscle fibres of the diaphragm in mice. Journal of Electron Microscopy 49:531-538

31. Ostrander EA, Huson HJ, Ostrander GK (2009) Genetics of Athletic Performance Annu. Rev. Genomics Hum Genet 10:407429

32. Mills CD, Ley K (2014) M1 and M2 macrophages: the chicken and the egg of immunity. J Innate Immun 6:716-726

33. Nahrendorf M, Swirski FK (2013) Monocyte and Macrophage Heterogeneity in the Heart. Circulation Research 112:1624-1633

34. D'Ascenzo S, Millimaggi D, Di Massimo C, Saccani-Jotti G, Botrè F, Carta G, Tozzi-Ciancarelli MG, Pavan A, Dolo V (2007) Detrimental effects of anabolic steroids on human endothelial cells. Toxicology Letters 169:129-136

35. Riezzo I, De Carlo D, Neri M, Nieddu A, Turillazzi E, Fineschi V (2011) Heart disease induced by AAS abuse, using experimental mice/rats models and the role of exercise-induced cardiotoxicity. Mini Rev Med Chem 11:409-424

36. Frati P, Busardò F, Cipolloni L, Dominicis ED, Fineschi V (2015) Anabolic Androgenic Steroid (AAS) Related Deaths: Autoptic, Histopathological and Toxicological Findings Curr Neuropharmacol. 13(1):146-159 